\title{
ON THE GREEN'S FUNCTION FOR SECOND ORDER PARABOLIC DIFFERENTIAL EQUATIONS WITH DISCONTINUOUS COEFFICIENTS ${ }^{1}$
}

BY D. G. ARONSON

Communicated by Louis Nirenberg, April 15, 1963

1. Introduction. We consider the second order linear parabolic differential operator in divergence form

$$
L u \equiv \frac{\partial u}{\partial t}-\frac{\partial}{\partial x_{j}}\left\{a_{i j}(x, t) \frac{\partial u}{\partial x_{i}}\right\}
$$

for $(x, t) \in Q_{T} \equiv \Omega \times(0, T]$, where $\Omega$ is a bounded open simply connected region in $E^{n}(n \geqq 2), T$ an arbitrary positive number, and the $a_{i j}$ are assumed only to be bounded and measurable in $\bar{Q}_{T}$. The purpose of this note is to report results concerning the existence and certain properties of the generalized Green's function $g\left(x_{0}, t_{0} ; x, t\right)$ for $L$ subject to homogeneous boundary conditions. Our results are largely based on the maximum principle for the problem

$$
\begin{aligned}
L u & =\operatorname{div} f(x, t)-f(x, t) \text { in } Q_{T} ; \\
u(x, t) & =\psi(x, t) \text { on } \Gamma \equiv \bar{S} \cup(\Omega \times\{t=0\}),
\end{aligned}
$$

where $S \equiv \partial \Omega \times(0, T]$ and $f, \boldsymbol{f}, \psi$ are given functions. This maximum principle, which we discuss in $\S 2$, is a generalization of the maximum principle proved by Ladyženskaja and Ural'ceva [3] since we make less restrictive assumptions on the inhomogeneous terms in (1.1). In $\S 3$ we introduce the corresponding generalizations of the Hölder continuity, existence and uniqueness theorems of [3]. Our main results on the existence and properties of the Green's function are given in $\S \S 4,5$ and 6 . The proofs of the theorems which we state below, as well as extensions of our results to equations with lower order terms and to the case of nonzero boundary conditions will be given elsewhere. The author is indebted to Professor Guido Stampacchia who first introduced him to many of the ideas embodied in this work, and to Professor Hans Weinberger for many stimulating discussions in the course of its preparation.

2. The maximum principle. We shall always assume that the coefficients $a_{i j}$ of $L$ are measurable in $Q_{T}$ and that there exists a constant

1 This work was supported in part by the Office of Naval Research under Contract Nonr-710(16), (NR 043 041). 
$\nu>0$ such that

$$
\nu^{-1}|\xi|^{2} \leqq a_{i j}(x, t) \xi_{i} \xi_{j} \leqq \nu|\xi|^{2} \text { a.e. in } \bar{Q}_{T}
$$

for all real vectors $\xi$. If $f(x) \in L^{\alpha}(\Omega)$ we shall write $\|f\|_{\alpha}$ for the $L^{\alpha}(\Omega)$ norm of $f$ and if ${ }^{2} f(x, t) \in L^{\beta}\left[0, T ; L^{\alpha}(\Omega)\right]$ we shall write $\|f\|_{\alpha}(t)$ for the $L^{\alpha}(\Omega)$-norm of $f$ and

$$
\|f\|_{\alpha, \beta}=\left\{\int_{0}^{T}\|f\|_{\alpha}^{\beta}(t) d t\right\}^{1 / \beta}
$$

THEOREM 1. Let $u$ be a smooth solution of (1.1) in $\bar{Q}_{T}$, where $f \in L^{s}\left[0, T ; L^{r}(\Omega)\right]$ for $n / 2 r+1 / s<1$ and $\boldsymbol{f}=\left(f_{1}, \cdots, f_{n}\right)$ with $f_{i} \in L^{q}\left[0, T ; L^{p}(\Omega)\right]$ for $n / 2 p+1 / q<1 / 2 .^{3}$ There exists a constant $K>0$ which depends only on $n, p, q, r, s, \nu$ and $|\Omega|$ such that

$$
\min _{\mathbf{r}} \psi-K F \leqq u(x, t) \leqq \max _{\mathbf{r}} \psi+K F
$$

in $\bar{Q}_{T}$, where $F=\|f\|_{r, s}+\sum_{i}\left\|f_{i}\right\|_{p, q}$. If $f=f(x) \in L^{r}(\Omega)$ for $r>n / 2$ and $f_{i}=f_{i}(x) \in L^{p}(\Omega)$ for $p>n$ then (2.2) holds with $F=\|f\|_{r}+\sum_{i}\left\|f_{i}\right\|_{p}$ and $K$ depending only on $n, p, r, \nu$ and $|\Omega|$.

The proof of Theorem 1 is based on the differential inequality

$$
\begin{aligned}
I_{k}^{\prime}(t)+ & C\left|A_{k}(t)\right|^{-2 / n} I_{k}(t) \\
& \leqq C\left\{\left|A_{k}(t)\right|^{1-2 / r^{*}}\|f\|_{r}^{2}(t)+\left|A_{k}(t)\right|^{1-2 / p} \sum_{i}\left\|f_{i}\right\|_{p}^{2}(t)\right\},
\end{aligned}
$$

where $1 / r^{*}=1 / r-1 / n, A_{k}(t)=\{x \mid x \in \Omega, u(x, t)>k\}$ and

$$
I_{k}(t)=\int_{A_{k}(t)}(u-k)^{2} d x .
$$

Here and for the remainder of this paper we adopt the convention that $C$ denotes any constant which depends only on $n, p, q, r, s, \nu$ and $|\Omega|$. The inequality (2.3) holds at all $t$ for which $I_{k}(t) \neq 0$ and for all $k \geqq \max \psi$. Integrating (2.3) and using the fact that $I_{k}(0)=0$, we obtain the estimate $I_{k}(t) \leqq C\left(J_{0}+\sum_{i} J_{i}\right)$, where, for example,

$$
J_{0}=\int_{r}^{t}\left|A_{k}(\eta)\right|^{1-2 / r^{*}}\|f\|_{r}^{2}(\eta) \exp \left[-C \int_{\eta}^{t}\left|A_{k}(y)\right|^{-2 / n} d y\right] d \eta
$$

for some $\tau \in[0, t)$. From this we deduce the estimate

2 I.e., $f$ is a function on $(0, T)$ to $L^{\alpha}(\Omega)$ which belongs to $L^{\beta}(0, T)$.

${ }^{8}$ In [3] only the case $s=q=+\infty$ is considered. 


$$
I_{k}(t) \leqq C F^{2}\left\{\max _{[0, T]}\left|A_{k}(\eta)\right|\right\}^{\alpha}
$$

where

$$
\alpha=\min \left\{1-\frac{2}{s}+\frac{2}{n}\left(1-\frac{2}{r^{*}}\right), 1-\frac{2}{p}+\frac{2}{n}\left(1-\frac{2}{q}\right)\right\}>1 .
$$

The proof of the right-hand side of (2.4) is now completed by noting that $(h-k)^{2}\left|A_{h}(t)\right| \leqq I_{k}(t)$ for $h>k$ and applying a lemma due to Stampacchia [7].

3. Hölder continuity and existence. The next theorem is a generalization of the corresponding result in [3]. The proof of our result is similar to the proof presented in [3] with suitable modifications to accommodate our broader assumptions on $f$ and $f$.

Theorem 2. Let $Q$ be such that $\bar{Q} \subset Q_{T}$ and let $\delta$ be the distance from $Q$ to $\Gamma$. Under the hypothesis of Theorem 1 , there exist constants $\alpha \in(0,1)$ and $C_{F, \delta}>0$ such that

$$
|u|_{\alpha, Q} \equiv \max _{Q} \frac{\left|u(x, t)-u\left(x^{\prime}, t^{\prime}\right)\right|}{\left\{\left|x^{\prime}-x\right|^{2}+\left|t^{\prime}-t\right|\right\}^{\alpha / 2}} \leqq C_{F, \delta},
$$

where $\alpha$ depends on $\delta, F, n, p, q, r, s, \nu$ and $|\Omega|$. If $\partial \Omega$ satisfies condition (A) of [3] and $|\psi|_{\beta, \Gamma} \leqq \gamma$, then

$$
|u|_{\alpha, Q_{T}} \leqq C,
$$

where $C$ and $\alpha$ depend on $\beta, \gamma$ and $\partial \Omega$ as well as $F, n, p, q, r, s, \nu$ and $|\Omega|$.

We shall now restrict our attention to the case $\psi \equiv 0$ on $\bar{S}$. We shall say that $u=u(x, t)$ is a generalized solution of problem (1.1) in $Q_{T}$ if $u \in L^{\infty}\left[0, T ; L^{2}(\Omega)\right] \cap L^{2}\left[0, T ; H_{0}^{1,2}(\Omega)\right]$ and satisfies

$$
\begin{aligned}
\int_{\Omega} u(x, t) \phi(x, t) d x & +\int_{0}^{t} d \tau \int_{\Omega}\left\{-u \phi_{t}+a_{i j} u_{x_{i}} \phi_{x_{i}}+f_{i} \phi_{x_{i}}+f \phi\right\} d x \\
& =\int_{\Omega} \psi(x) \phi(x, 0) d x
\end{aligned}
$$

for all $t \in(0, T]$ and for all $\phi \in C^{\prime}\left(\bar{Q}_{T}\right)$ with compact support in $\Omega$ (test functions).

TheOREM 3. If $\psi \in L^{2}(\Omega)$ and if the inhomogeneous terms satisfy the hypothesis of Theorem 1 , then there exists a generalized solution $u=u(x, t)$ of (1.1) (with $\psi \equiv 0$ on $\bar{S}$ ) which is Hölder continuous on every compact 
interior subset of $Q_{T}$. Moreover, $u$ is unique in the class $L^{\infty}\left[0, T ; L^{2}(\Omega)\right]$ $\cap L^{2}\left[0, T ; H_{0}^{1,2}(\Omega)\right]$. If $\psi$ is bounded on $\bar{\Omega}$, then the maximum principle (2.2) holds for $u$. If $\psi \in C_{0}^{\beta}(\Omega)$ and $\partial \Omega$ satisfies condition (A), then $u$ is Hölder continuous in $\bar{Q}_{T}$.

We note that even in the case $f, f_{i} \equiv 0$ the class of generalized solutions which we defined above does not always coincide with the class of generalized solutions studied in [3]. In particular, the solutions in [3] belong to $L^{\infty}\left[0, T ; L^{2}(\Omega)\right] \cap L^{2}\left[0, T ; W^{1,2}(\Omega)\right]$ and satisfy (3.2) for all $\phi \in W^{1,2}\left(Q_{T}\right)$ such that $\phi=0$ on $\bar{S}$. It is not difficult to give sufficient conditions for the coincidence of these two classes of solutions.

4. Existence of the Green's function. Let $\mu=(r, s, p, q)$ and $\mu^{\prime}=\left(r^{\prime}, s^{\prime}, p^{\prime}, q^{\prime}\right)$, where $1 / r+1 / r^{\prime}=1$, etc. We shall say that $\mu$ is admissible if $n / 2 r+1 / s<1$ and $n / 2 p+1 / q<1 / 2$. Let

$\mathcal{L}^{\mu^{\prime}}\left(Q_{T}\right)=L^{s^{\prime}}\left[0, T ; L^{r^{\prime}}(\Omega)\right] \oplus L^{q^{\prime}}\left[0, T ; L^{p^{\prime}}(\Omega)\right] \oplus \cdots \oplus L^{q^{\prime}}\left[0, T ; L^{p^{\prime}}(\Omega)\right]$.

If $h=\left(h_{0}, h_{1}, \cdots, h_{n}\right) \in \mathcal{L}^{\mu^{\prime}}\left(Q_{T}\right)$ we define

$$
\|h\|_{\mu^{\prime}}=\left\|h_{0}\right\|_{r^{\prime}, 8^{\prime}}+\sum_{i}\left\|h_{i}\right\|_{p^{\prime}, q^{\prime}}
$$

With this norm $\mathscr{L}^{\mu^{\prime}}\left(Q_{T}\right)$ is a Banach space. Moreover $\left\{\mathscr{L}^{\mu^{\prime}}\left(Q_{T}\right)\right\}^{*}$ $=\mathscr{L}^{\mu}\left(Q_{T}\right)$ and $\left\{\mathcal{L}^{\mu^{\prime}}\left(Q_{T}\right)\right\}^{* *}=\mathscr{L}^{\mu^{\prime}}\left(Q_{T}\right)$ [1]. Let $H_{0}^{1, \mu^{\prime}}\left(Q_{T}\right)$ be the closure in $\mathcal{L}^{\mu^{\prime}}\left(Q_{T}\right)$ of vectors of the form $\left(\phi, \operatorname{grad}_{x} \phi\right)$, where $\phi$ is a test function. Define $H^{-1, \mu^{\prime}}\left(Q_{T}\right) \equiv\left\{H_{0}^{1, \mu^{\prime}}\left(Q_{T}\right)\right\}^{*}$. Then $H^{-1, \mu^{\prime}}\left(Q_{T}\right)$ is equivalent to $\mathscr{L}^{\mu}\left(Q_{T}\right) / \mathscr{T}^{\mu}\left(Q_{T}\right)$, where $\mathfrak{T}^{\mu}\left(Q_{T}\right)$ is the class of all $h \in \mathcal{L}^{\mu}\left(Q_{T}\right)$ which satisfy

$$
\int_{0}^{T} d t \int_{\Omega} h \cdot\left(\phi, \operatorname{grad}_{x} \phi\right) d x=0
$$

for all test functions $\phi$.

Consider (1.1) for $\psi \equiv 0$ and $h=(-f, f) \in \mathcal{L}^{\mu}\left(Q_{T}\right)$ for an admissible $\mu$. Let $\left(x_{0}, t_{0}\right)$ be a fixed point in $Q_{T}$. It follows from the maximum principle and (3.2) that the value of the generalized solution of (1.1) at $\left(x_{0}, t_{0}\right)$ is a bounded linear functional on $H^{-1, \mu^{\prime}}\left(Q_{T}\right)$, i.e., $u\left(x_{0}, t_{0}\right)$ $=\mathcal{G}(\boldsymbol{h})$, where $\mathcal{G} \in\left\{H^{-1, \mu^{\prime}}\left(Q_{T}\right)\right\}^{*}=H_{0}^{1, \mu^{\prime}}\left(Q_{T}\right)$. This, together with similar considerations in the case $h$ independent of $t$ lead to

Theorem 4 . Let $\boldsymbol{h}=(-f, \boldsymbol{f}) \in \mathcal{L}^{\mu}\left(Q_{T}\right)$ for an admissible $\mu$ and $\psi \equiv 0$. For any $\left(x_{0}, t_{0}\right) \in Q_{T}$ the generalized solution of (1.1) can be written in the form

$$
u\left(x_{0}, t_{0}\right)=\int_{0}^{T} d t \int_{\Omega} \boldsymbol{g}\left(x_{0}, t_{0} ; x, t\right) \cdot \boldsymbol{h}(x, t) d x
$$


where $\boldsymbol{g}=\left(g, \operatorname{grad}_{x} g\right) \in H_{0}^{1, \mu^{\prime}}\left(Q_{T}\right)$ as a function of $(x, t)$. Moreover, the generalized Green's function $g$ is unique and

$$
\left\|\boldsymbol{g}\left(x_{0}, t_{0} ; \cdot, \cdot\right)\right\|_{\mu^{\prime}} \leqq C
$$

in $\bar{Q}_{T}$ for every admissible $\mu$. If $\boldsymbol{h}$ is independent of $t$ then

$$
u\left(x_{0}, t_{0}\right)=\int_{\Omega}\left\{\int_{0}^{T} \xi\left(x_{0}, t_{0} ; x, t\right) d t\right\} \cdot h(x) d x
$$

where

$$
\left\|\int_{0}^{T} g\left(x_{0}, t_{0} ; \cdot, t\right) d t\right\|_{L^{r^{\prime}}(\Omega)}+\left\|\int_{0}^{T} g\left(x_{0}, t_{0} ; \cdot, t\right) d t\right\|_{H_{0}^{1, p^{\prime}}(\Omega)} \leqq C
$$

for all $r>n / 2$ and $p>n$.

If we approximate $\Omega$ by an increasing sequence of smooth domains $\left\{\Omega^{m}\right\}$ for $m=1,2, \cdots$ and mollify the coefficients and data in (1.1), it follows from results of Pogorzelski [6] that the solutions of the resulting smooth problem can be written in the form

$$
u^{m}\left(x_{0}, t_{0}\right)=\int_{0}^{t_{0}} d t \int_{\Omega^{m}} \boldsymbol{g}^{m}\left(x_{0}, t_{0} ; x, t\right) \cdot \boldsymbol{h}^{m}(x, t) d x .
$$

Using Theorem 3 we conclude that $u^{m} \rightarrow u$ and we obtain

Theorem 5. Let $Q$ be such that $\bar{Q} \subset Q_{T}$. For all $\left(x_{0}, t_{0}\right) \in \bar{Q}, g^{m} \rightarrow g$ weakly in $H_{0}^{1, \mu^{\prime}}\left(Q_{T}\right)$ for any admissible $\mu, \int_{0}^{t_{0}} g^{m} d t \rightarrow \int_{0}^{T} g d t$ weakly in $H_{0}^{1, p^{\prime}}(\Omega)$ for $p>n$, and $\int_{0}^{t_{0}} g^{m} d t \rightarrow \int_{0}^{T} g d t$ strongly in $L^{a}(\Omega)$ for any $a<n /(n-2)$. If $\partial \Omega$ satisfies condition (A) we can take $Q \equiv Q_{T}$.

From Theorem 5 we conclude that $g \geqq 0$ and $g\left(x_{0}, t_{0} ; x, t\right) \equiv 0$ for $t>t_{0}$.

5. Properties of the Green's function. Using known properties of the Green's function in the smooth case [6] and a slight modification of an estimate due to Nash [5] we obtain

Theorem 6. Let $\Omega^{\prime}$ be such that $\bar{\Omega}^{\prime} \subset \Omega$ and let $\hat{t}>0$. As a function of $(x, t)$ the Green's function $g(x, t ; \xi, 0)$ of $L$ is Hölder continuous in $\bar{\Omega}^{\prime} \times[\hat{t}, T]$ and satisfies

$$
\begin{aligned}
\int_{\Omega} g(x, t ; \xi, 0) \phi(x, t) d x+\int_{\hat{t}}^{t} d \tau \int_{\Omega}\left\{-g \phi_{\imath}+a_{i j} g_{x_{i}} \phi_{x_{j}}\right\} d x \\
\quad=\int_{\Omega} g(x, \hat{t} ; \xi, 0) \phi(x, \hat{t}) d x
\end{aligned}
$$


for all test functions $\phi$. Moreover, $g$ is the uniform limit of $\left\{g^{m}\right\}$ in $\bar{\Omega}^{\prime} \times[\hat{t}, T]$, the weak limit $\left\{g^{m}\right\}$ in $L^{2}\left[\hat{t}, T ; H_{0}^{1,2}(\Omega)\right]$, and

$$
\int_{\Omega} g^{2}(x, t ; \xi, 0) d x+\frac{1}{\nu} \int_{\hat{t}}^{t} d \tau \int_{\Omega}\left|\operatorname{grad}_{x} g\right|^{2} d x \leqq C \hat{t}^{-n / 2} .
$$

If $\partial \Omega$ satisfies condition (A) we can take $\Omega^{\prime} \equiv \Omega$.

From Theorems 5 and 6 we can prove

Theorem 7. Let $f, f \equiv 0, \psi \equiv 0$ on $\bar{S}$ and $\psi \in L^{2}(\Omega)$. Then for $(x, t) \in Q_{T}$ the generalized solution of (1.1) is given by

$$
u(x, t)=\int_{\Omega} g(x, t ; \xi, 0) \psi(\xi) d \xi .
$$

In particular, it follows from Theorems 4 and 7 that the usual representation formula holds for the generalized solution of (1.1) in the case $\psi \equiv 0$ on $\bar{S}$.

6. Asymptotic behavior. Consider the problem

$$
\begin{aligned}
L u & =\operatorname{div} f(x) \text { in } \Omega \times\{t>0\} ; \\
u & =0 \text { on }(\partial \Omega \times\{t \geqq 0\}) \cup(\Omega \times\{t=0\})
\end{aligned}
$$

where $f_{i} \in L^{p}(\Omega)$ for $p>n, a_{i j}(x, t) \rightarrow \bar{a}_{i j}(x)$ as $t \rightarrow \infty$ uniformly for $x \in \bar{\Omega}$, and (2.1) holds a.e. in $\bar{\Omega} \times[0, \infty]$. It follows from the results of Littman, Stampacchia and Weinberger [4] that the corresponding steady state problem

$$
\left\{\bar{a}_{i j}(x) v_{x_{i}}\right\}_{x_{j}}=-\operatorname{div} f(x) \text { in } \Omega ; \quad v=0 \text { on } \partial \Omega
$$

has a unique generalized solution which is given by

$$
v\left(x_{0}\right)=\int_{\mathbf{\Omega}} \operatorname{grad}_{x} G\left(x_{0}, x\right) \cdot f(x) d x,
$$

where $G \in H_{0}^{1, p^{\prime}}(\Omega)$ for any $p>n$. Combining this with our results and a result of Friedman's [2] on the steady state behavior of solutions of parabolic equations in the smooth case we obtain

Theorem 8. Let $\Omega^{\prime}$ be such that $\bar{\Omega}^{\prime} \subset \Omega$. Then $u(x, t) \rightarrow v(x)$ as $t \rightarrow \infty$ uniformly for $x \in \bar{\Omega}^{\prime}, \int_{0}^{t} g\left(x_{0}, t ; x, \tau\right) d \tau \rightarrow G\left(x_{0}, x\right)$ as $t \rightarrow \infty$ weakly in $H_{0}^{1, p^{\prime}}(\Omega)$ as a function of $x$ for $x_{0} \in \Omega$, and $\int_{0}^{t} g d \tau \rightarrow G$ as $t \rightarrow \infty$ strongly in $L^{a}(\Omega)$ for any $a<n /(n-2)$. 


\title{
REFERENCES
}

1. S. Bochner and A. E. Taylor, Linear functionals on certain spaces of abstractlyvalued functions, Ann. of Math. (2) 39 (1938), 913-944.

2. Avner Friedman, Convergence of solutions of parabolic equations to a steady state, J. Math. Mech. 8 (1959), 57-76.

3. O. A. Ladyženskaja and N. N. Ural'ceva, $A$ boundary-value problem for linear and quasi-linear parabolic equations. I, Izv. Akad. Nauk SSSR Ser. Mat. 26 (1962), 5-52. (Russian)

4. W. Littman, G. Stampacchia and H. F. Weinberger, Regular points for elliptic equations with discontinuous coeficients, University of Minnesota Report, Minneapolis, Minn., December 1962.

5. J. Nash, Continuity of solutions of parabolic and elliptic equations, Amer. J. Math. 80 (1958), 931-954.

6. W. Pogorzelski, Etude d'une fonction de Green et du probleme aux limites pour l'équation parabolique normale, Ann. Polon. Math. 4 (1958), 288-307.

7. G. Stampacchia, Régularisation des solutions de problèmes aux limites elliptiques a données discontinues, Internat. Sympos. on Linear Spaces, Pergamon, Jerusalem, 1960.

UNIVERSITY OF MinNESOTA

\section{ON APPROXIMATE SOLUTIONS TO THE CONVOLUTION EQUATION ON THE HALF-LINE}

\author{
BY T. K. BOEHME
}

Communicated by R. C. Buck, July 24, 1963

1. We will call a complex-valued function on the half-line $t>0$ locally integrable if it is integrable on each interval $[0, T], T>0$. Let $\&$ be the ring of locally integrable functions (functions which are equal up to a set of measure zero will be identified with each other) with the usual pointwise addition, and with convolution for the product operation. Thus $k x=r$ if and only if $\int_{0}^{t} k(t-u) x(u) d u=r(t)$ for almost every $t>0$. Give $\&$ the topology defined by the seminorms $\|x\|_{T}=\int_{0}^{T}|x|(u) d u, T>0$. Thus a sequence $x_{n}, n=1,2, \cdots$ in $\&$ converges to 0 in $\&$ if and only if $x_{n} \rightarrow 0$ in $L[0, T]$ for each $T>0$ as $n \rightarrow \infty$. The equation $k x=r$ is an important integral equation; however, solutions and the existence of solutions are in general difficult to obtain. M. I. Fenyö and C. Foias [1] ${ }^{1}$ have shown that if $k$ and $r$ are in $\mathscr{L}$ and if $k$ vanishes on no neighborhood of the origin (i.e. $\|k\|_{T}>0$ for each $T>0$ ) there is always an approximate solution to the equation

1 The author thanks the referee for calling this article to his attention. 